\title{
Archéopages
}

Archéopages

Archéologie et société

42 | 04-07/2015

Construire en terre crue

\section{Constructions mixtes en terre et bois}

Un village $\mathrm{du}^{\mathrm{e}}$ siècle avant notre ère en Bourgogne

Mixed earth and timber structures. A fifth-century BC village in Burgundy

Construcciones mixtas de barro y madera. Una aldea del siglo $V$ antes de nuestra

era en Borgoña

Régis Labeaune, Christophe Gaston et Dominique Sordoillet

\section{(2) OpenEdition}

\section{Journals}

Édition électronique

URL : https://journals.openedition.org/archeopages/1166

DOI : 10.4000/archeopages. 1166

ISSN : 2269-9872

Éditeur

INRAP - Institut national de recherches archéologiques préventives

Édition imprimée

Date de publication : 1 mars 2016

Pagination : 36-47

ISSN : 1622-8545

\section{Référence électronique}

Régis Labeaune, Christophe Gaston et Dominique Sordoillet, «Constructions mixtes en terre et bois », Archéopages [En ligne], 42 | 04-07/2015, mis en ligne le 01 juillet 2017, consulté le 04 juin 2021. URL : http://journals.openedition.org/archeopages/1166; DOI : https://doi.org/10.4000/archeopages. 1166 


\section{Constructions mixtes en terre et bois Un village du v viècle avant notre ère en Bourgogne}

Régis Labeaune Inrap, UMR 6298, "ARTTHIS"

Christophe Gaston IIrrap, UMR 6249, “Chrono-environnement"

Dominique Sordoillet Inrap, UMR 624, «Chrono-environement "

36

1

1

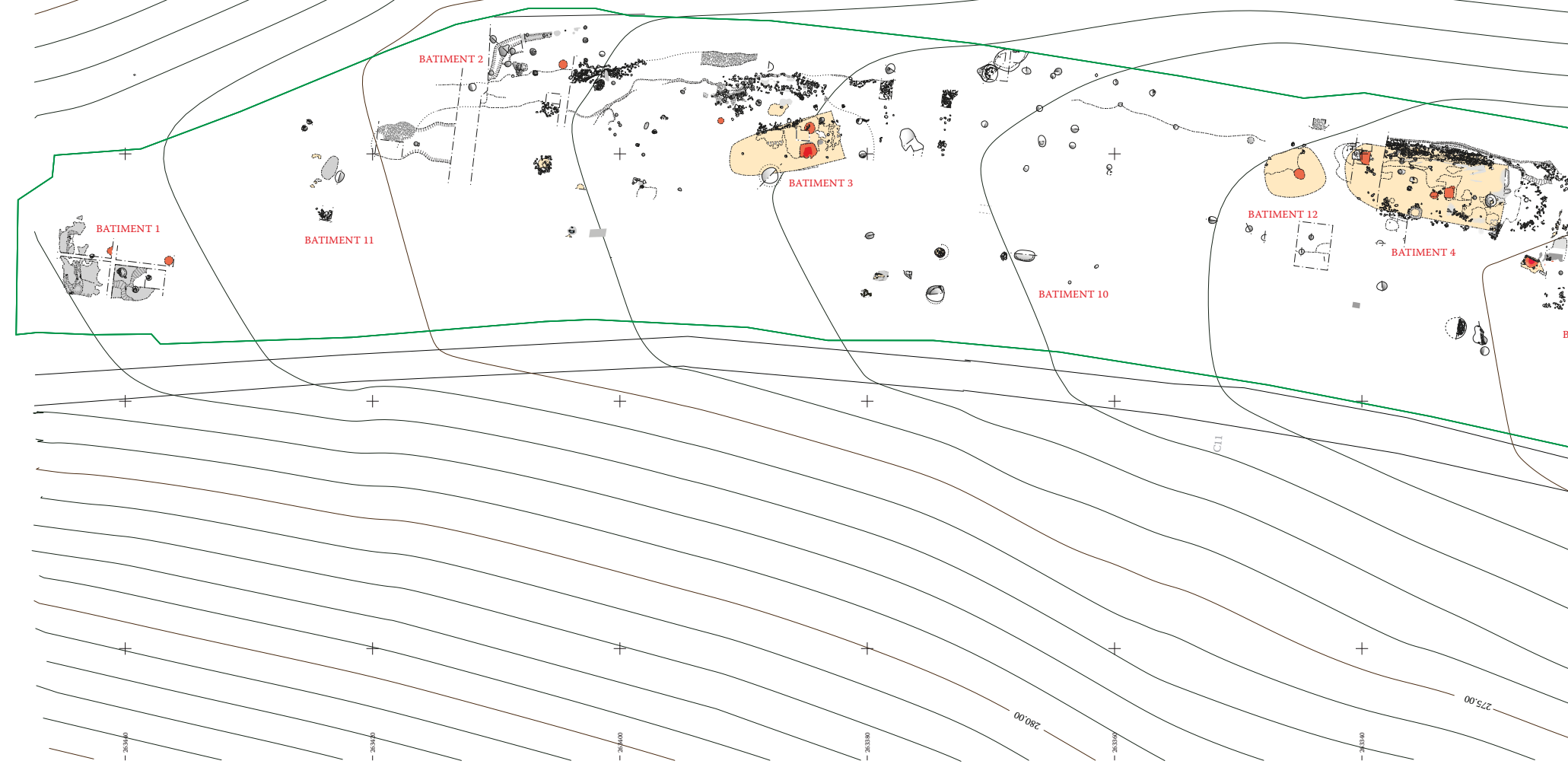


1. Fouille réalisée sur une surface de 8 ooo $\mathrm{m}^{2}$ en 2009, sous la direction de Régis Labeaune, Inrap.
À l'ouest de Dijon, la fouille de « la Peute Combe » à Talant (Côte-d'Or) a mis au jour quatorze bâtiments de la fin du premier âge du Fer, pour la plupart à abside $\mathbf{1}$ (Labeaune, Alix, 2014). Au centre de cet habitat, un atelier polymétallique, spécialisé dans la fabrication de petits objets en fer et en bronze, avait conservé ses foyers et ses aires de travail. Il a donc été possible de restituer un plan et une organisation du village. La datation du site d'après le mobilier métallique est homogène car la majeure partie des objets sont datés de la première moitié $d u v^{e}$ siècle avant notre ère. Après une cinquantaine d'années d'occupation, le village est détruit, fort probablement par un incendie qui a laissé de nombreuses traces dans les couches supérieures de l'habitat. Le terrain est ensuite nivelé pour construire, à l'époque laténienne, une voie qui traverse la combe du nord au sud et qui a été utilisée pendant une centaine d'années. Cette structure a permis de protéger les niveaux archéologiques hallstattiens et nous avons pu observer la présence de sols en argile conservés au moins dans six bâtiments appartenant à la première occupation du site.

Le site est installé au fond d'un vallon étroit orienté nord-sud, dont les versants sont abrupts, incisant les plateaux calcaires et débouchant sur la vallée de l'Ouche. Les pentes sont caractérisées par une végétation arbustive et leur exposition ensoleillée donne à la vallée un aspect de paysage méditerranéen. L'érosion des pentes et le ruissellement constituent les facteurs principaux de l'accumulation de sédiments qui a permis une préservation exceptionnelle du site, ainsi protégé des labours ou de toute autre destruction des vestiges. Cette sédimentation atteint une épaisseur moyenne de 1,50 m entre les niveaux du $v^{\mathrm{e}}$ siècle avant notre ère et le sol actuel.

\section{La typologie des constructions}

L'organisation du village a été fortement conditionnée par le cadre topographique : la combe ne mesurant qu'une quarantaine de mètres de large, les constructions se sont installées dans sa longueur, formant ainsi un « village rue ». Quatorze maisons ont été identifiées lors de la fouille [ill. 1]. Leurs plans sont difficiles à observer car, même si la majorité des trous de poteaux porteurs subsistent grâce à leur profondeur, les cloisonnements internes et les murs périphériques restent lacunaires. Trois catégories de construction, qui se distinguent les

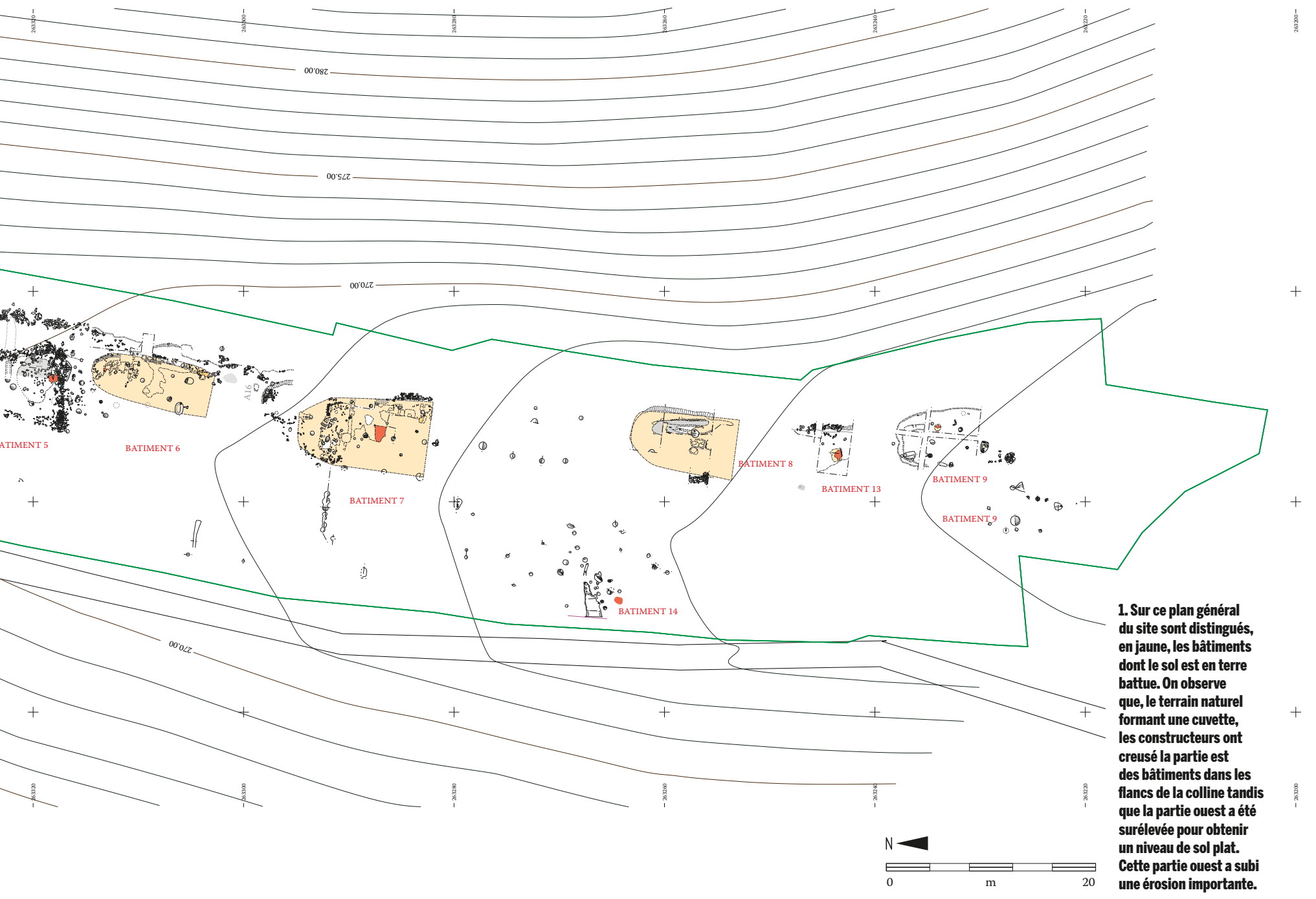


Bâtiments à plan allongé, de taille moyenne
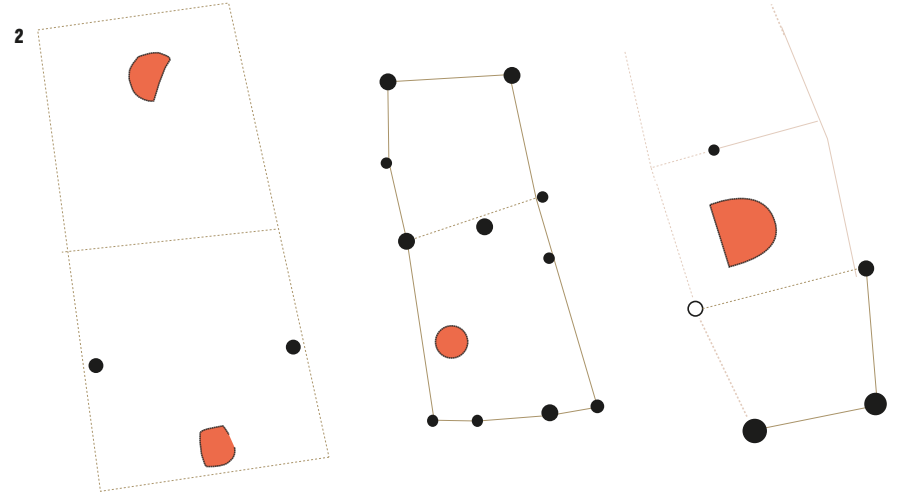

Bâtiments à plan centré

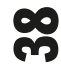

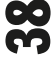

Bâtiments à plan allongé, de grande taille
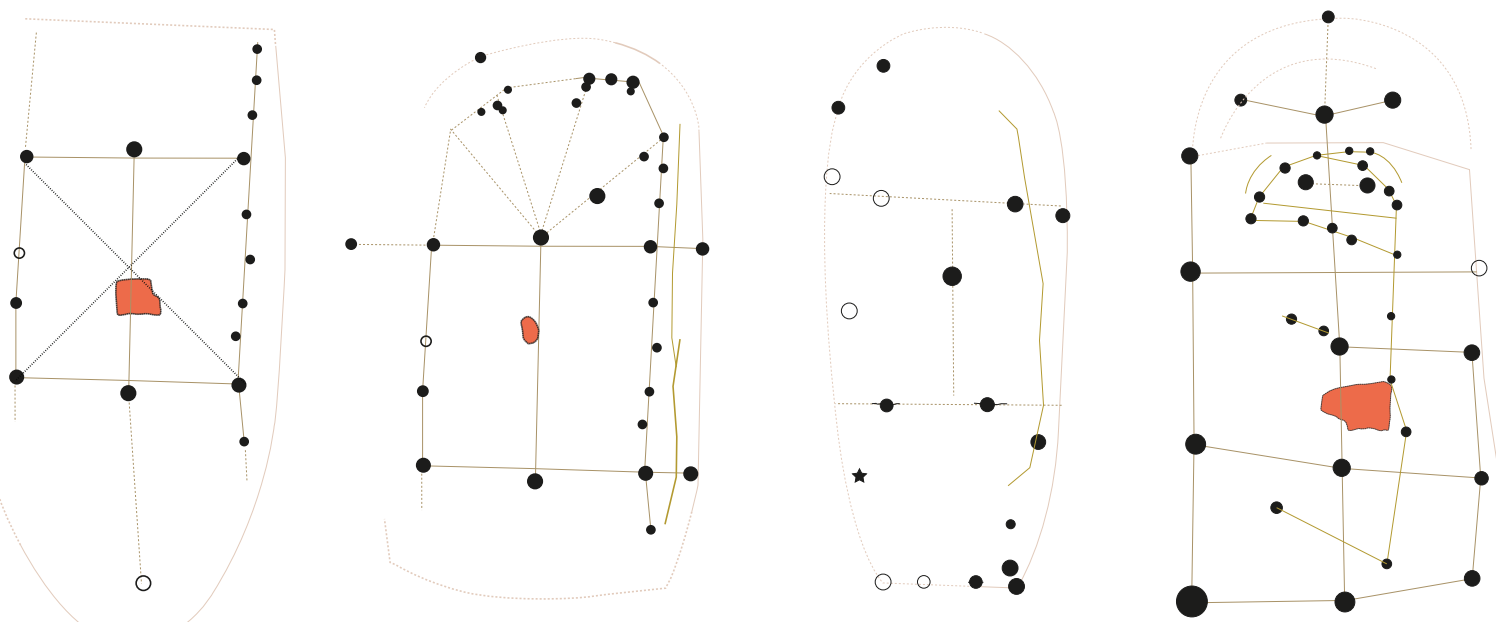
unes des autres par leurs dimensions et la nature de leurs sols, ont pu être identifiées [ill. 2].

Les bâtiments les plus grands mesurent environ 12 à 14 mètres de long pour 6 mètres de large. L'espace y est divisé en trois travées inégales. La travée centrale, abritant un foyer placé sur l'axe de symétrie, forme le châssis de base de toute la structure. Quatre poteaux en assurent l'ossature à portique, contre laquelle s'appuient deux poteaux faîtiers. Une abside, toujours située au nord, ferme le bâtiment, tandis qu'au sud une travée d'une largeur égale à la moitié de celle de la travée centrale pourrait soit recevoir une toiture en pavillon, soit former un « porche » couvert. La structure de construction est mixte, c'est-à-dire qu'elle associe des sablières porteuses pour les parois avec un encadrement de poteaux porteurs pour l'ossature interne. Les sols sont aménagés en argile damée.

Une deuxième catégorie de bâtiments est constituée de bâtiments de taille moyenne, qui présentent les mêmes proportions que les grands bâtiments, mais leur longueur est d'une dizaine de mètres environ et leur sol est aménagé avec des planchers dont il ne subsiste que les traces de lambourdes. Leur plan est plus lacunaire que ceux décrits précédemment, ce qui rend leur restitution difficile à interpréter.

Enfin, les bâtiments dont le plan rectangulaire est centré ; ils présentent un sol en « cuvette » de type fond de cabane. Un plancher est envisageable, en raison de la présence d'une couche charbonneuse sur toute la surface et, sous cette couche, d'une tranchée qui peut correspondre aux restes d'une lambourde. La structure de ce type de construction reste hypothétique : un poteau axial subsiste, décalé vers le nord, sans doute pour laisser l'espace nécessaire à un foyer central. Il est tentant de restituer un autre poteau axial, l'association des deux formant un portique qui aurait soutenu une faîtière centrale ; le bâtiment aurait ainsi été couvert par une toiture à quatre pans reposant sur les parois porteuses.

Ces différents bâtiments ont fait l'objet de plusieurs réfections ou de reconstructions successives et nous avons pu mettre en évidence trois états bien distincts pour au moins la moitié d'entre eux. Ces observations permettent de proposer l'idée que le village a été occupé pendant une bonne cinquantaine d'années avant d'être détruit par un incendie comme l'atteste la couche charbonneuse qui recouvre toutes les structures. Pour six de ces quatorze bâtiments, le sol était constitué d'une couche d'argile jaune.

\section{Le bâtiment 3}

Le plan du bâtiment 3 est très lacunaire mais deux états de construction sont déterminables [ill. 3]. Le plan rectangulaire du premier état est divisé en trois travées. Le sol en argile est construit sur un substrat qui a été épierré de ses plus gros éléments. La structure de l'ensemble est mixte, des sablières sur solins constitués de petits blocs, dont certains chauffés par un incendie, sont clairement identifiables pour la paroi est, limitant le sol en argile. Le foyer, positionné au centre de la deuxième travée du bâtiment, est disposé sur un léger relief constitué de gravier et de terre argileuse qui repose sur le sol du bâtiment. Le plan du second état est encore plus approximatif. Il pourrait correspondre à une reconstruction. Légèrement décalée vers l'est, une nouvelle couche d'argile « déborde » de l'axe de la sablière primitive et ne se prolonge pas au-delà du trou de poteau 2273. Son plan se divise en deux travées avec un foyer dans l'espace ouest. Les coupes stratigraphiques montrent clairement une superposition des sols et des foyers de ces deux états avec, entre les deux sols, une couche de limon grisâtre [ill. 4]. Les analyses micromorphologiques ont montré que le sol de la première occupation hallstattienne est composé d'un revêtement grisjaune, assez dense, d'argile et de micrite, avec des inclusions de sables et de cailloux calcaires. Dans le second état, un nouveau sol argilo-limoneux semble résulter de la dégradation de constructions en « terre ». Le matériel observé est assez différent des torchis souvent utilisés comme matériaux de construction (Sordoillet, 2009). L'absence de phytolithe d'herbacées dans les lames étudiées tend à exclure l'utilisation de paille et donc de torchis, et plaide plutôt pour la confection de murs à l'aide des nombreuses pierres présentes sur le site, liées entre elles par un mortier calcaire.

\section{Le bâtiment 4}

Le bâtiment 4 n'est conservé que sur une petite moitié est ; la fouille a montré qu'il a connu deux états successifs [ill. 5]. On aurait ici une architecture mixte associant des parois porteuses sur des sablières enterrées ou sur des murets et des supports verticaux internes. Cette technique, qui associe les sablières basses et les poteaux verticaux et porte le nom de Ständerbau, a été observée dans de nombreux bâtiments de l'époque, notamment celui de Pont-Rémy (18,40 m x 6,50 m) dans la Somme, daté de La Tène ancienne (Buchez, 2005). Les dimensions totales de ce bâtiment seraient d'environ $7 \mathrm{~m} \mathrm{x} 12 \mathrm{~m}$ pour le premier état, et de $7 \mathrm{~m} \times 13,5 \mathrm{~m}$ pour le deuxième. Ces dimensions sont proches de celles du bâtiment hallstattien (12 m x 6,50 m) d'Ennemain dans la Somme (Buchez, 2005), ou de celles du bâtiment hallstattien (14 $\mathrm{m} \mathrm{x} \mathrm{7,50} \mathrm{m)}$ de Barbey en Seine-et-Marne (Gouge, 2005).

Le premier bâtiment édifié offre un espace divisé en trois travées inégales. La travée centrale abrite un foyer placé sur son axe de symétrie. Le foyer, même s'il peut aussi se rencontrer adossé à une paroi, est souvent placé au centre du bâtiment (Curdy, 1993). Cette travée de plan carré (5 m x $5 \mathrm{~m}$ ) forme le châssis de base de toute la structure : quatre poteaux en assurent l'ossature à portique, contre laquelle deux poteaux faîtiers viennent s'appuyer, comme semble l'indiquer le léger décalage de ceux-ci. Ces constructions à faîtière porteuse impliquent que ce type d'ossature supporte une construction élaborée selon les axes 

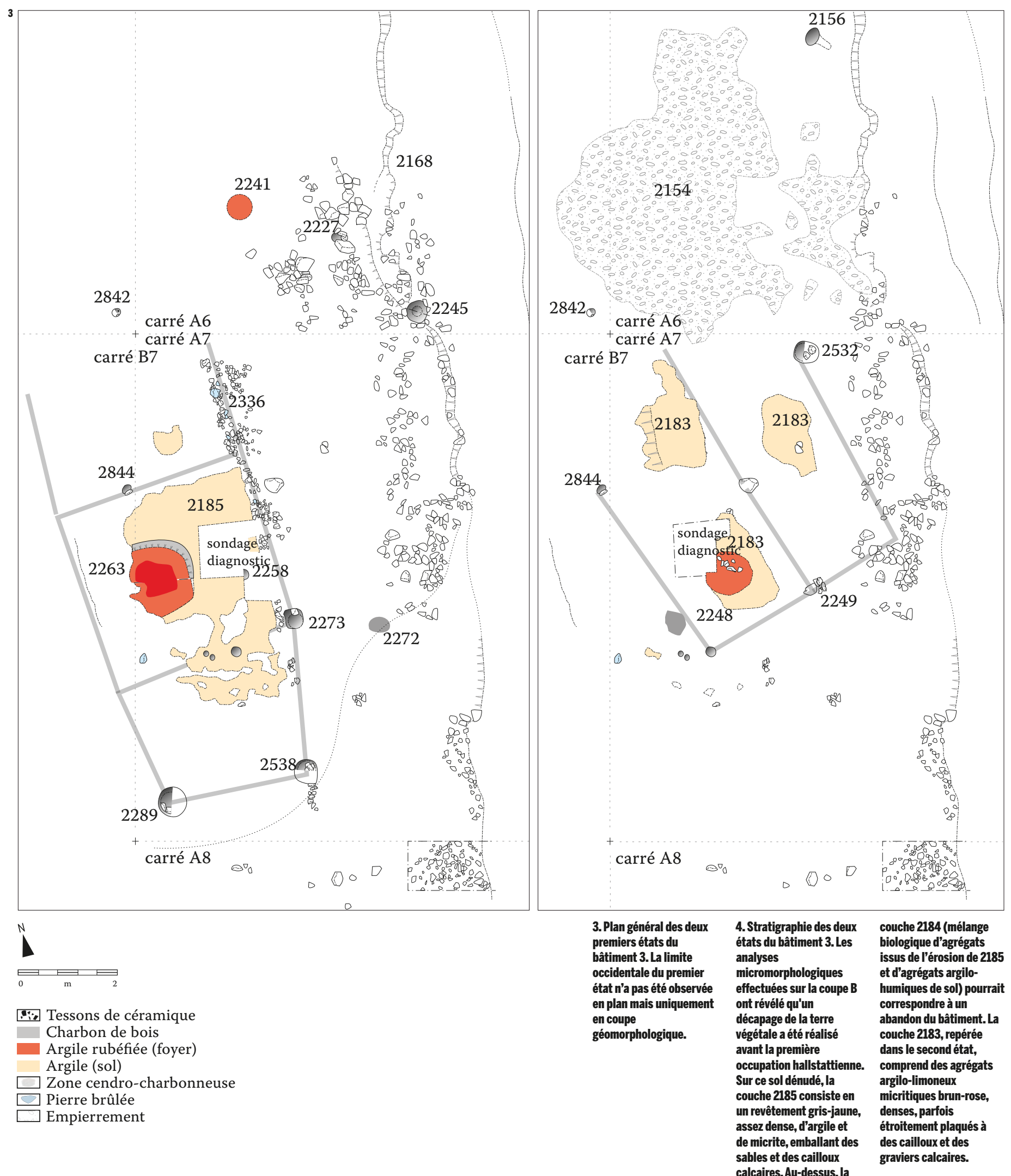

Tessons de céramique

Charbon de bois

Argile rubéfiée (foyer)

Argile (sol)

$\square$ Zone cendro-charbonneuse

$\checkmark$ Pierre brûlée

Empierrement 

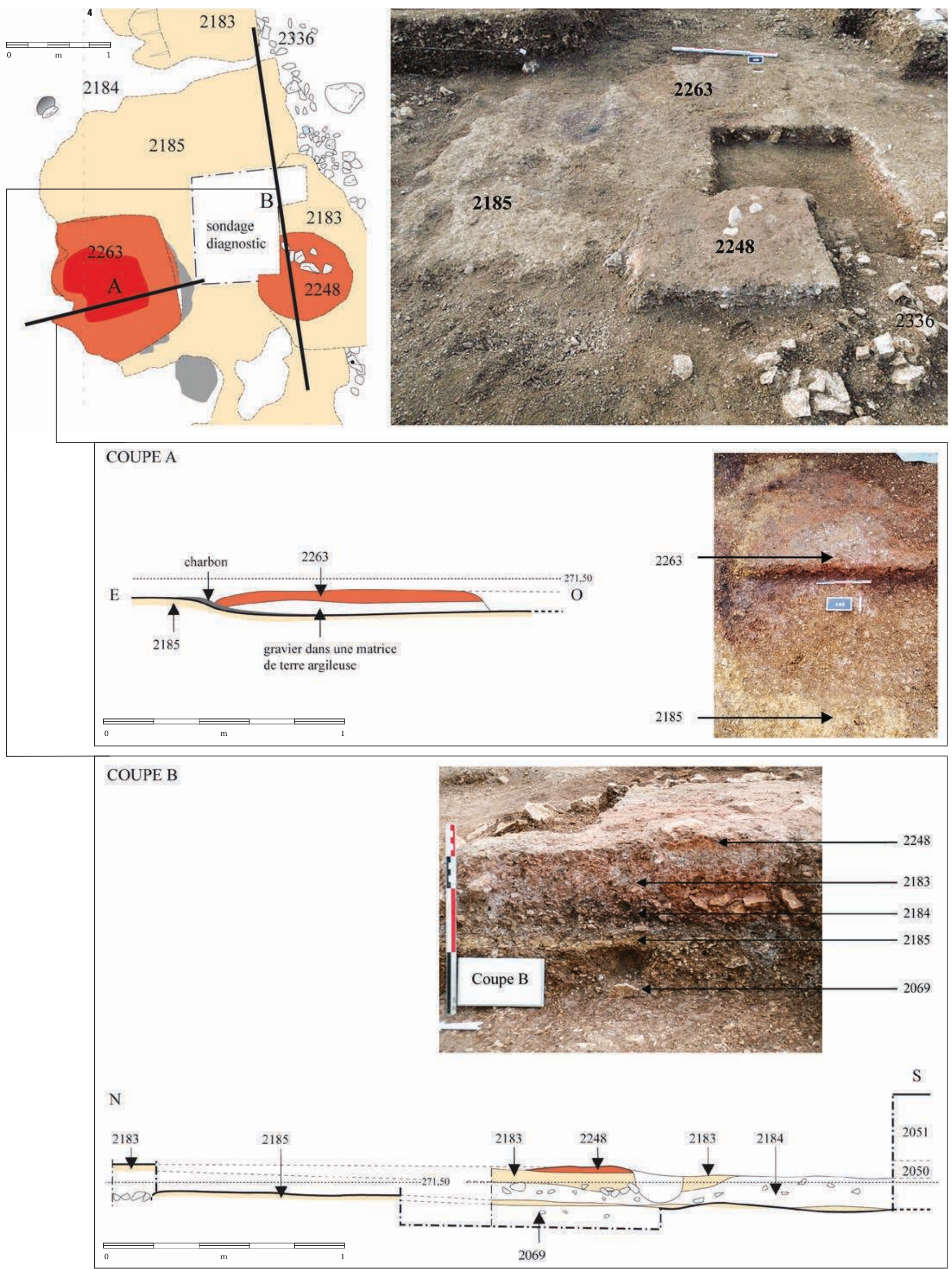

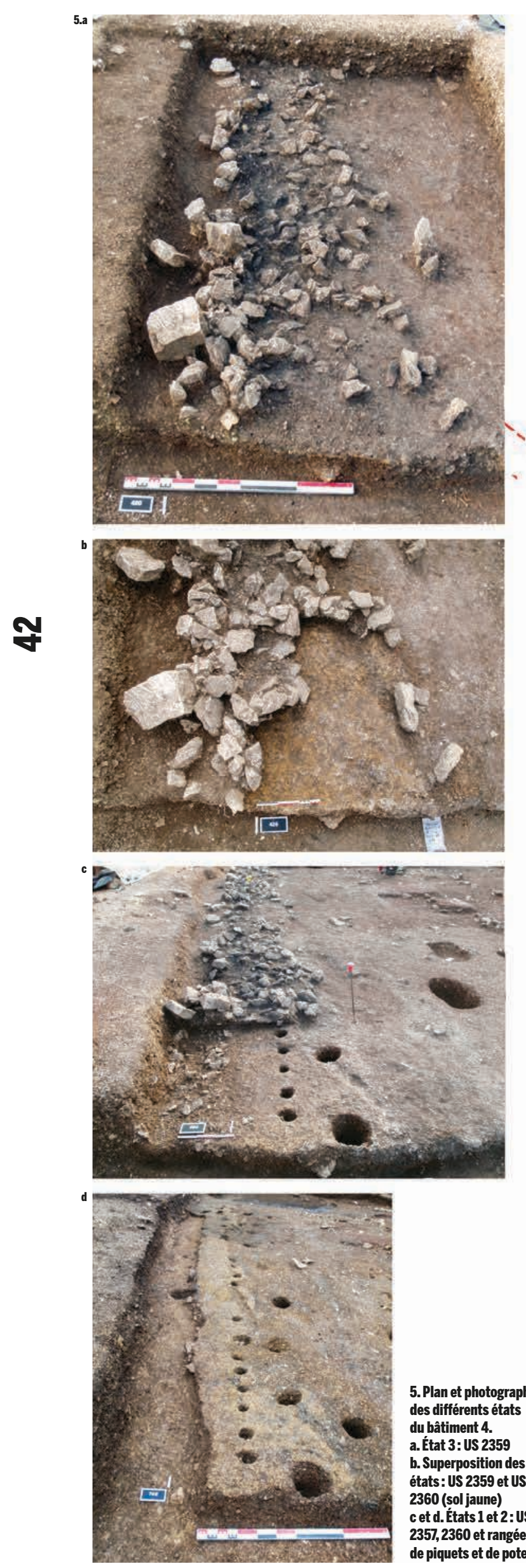

5. Plan et photographies des différents états du bâtiment 4. a. État 3: US 2359

b. Superposition des états: US 2359 et US 2360 (sol jaune)

cet d. États 1 et 2: US

2357, 2360 et rangées

de piquets et de poteaux

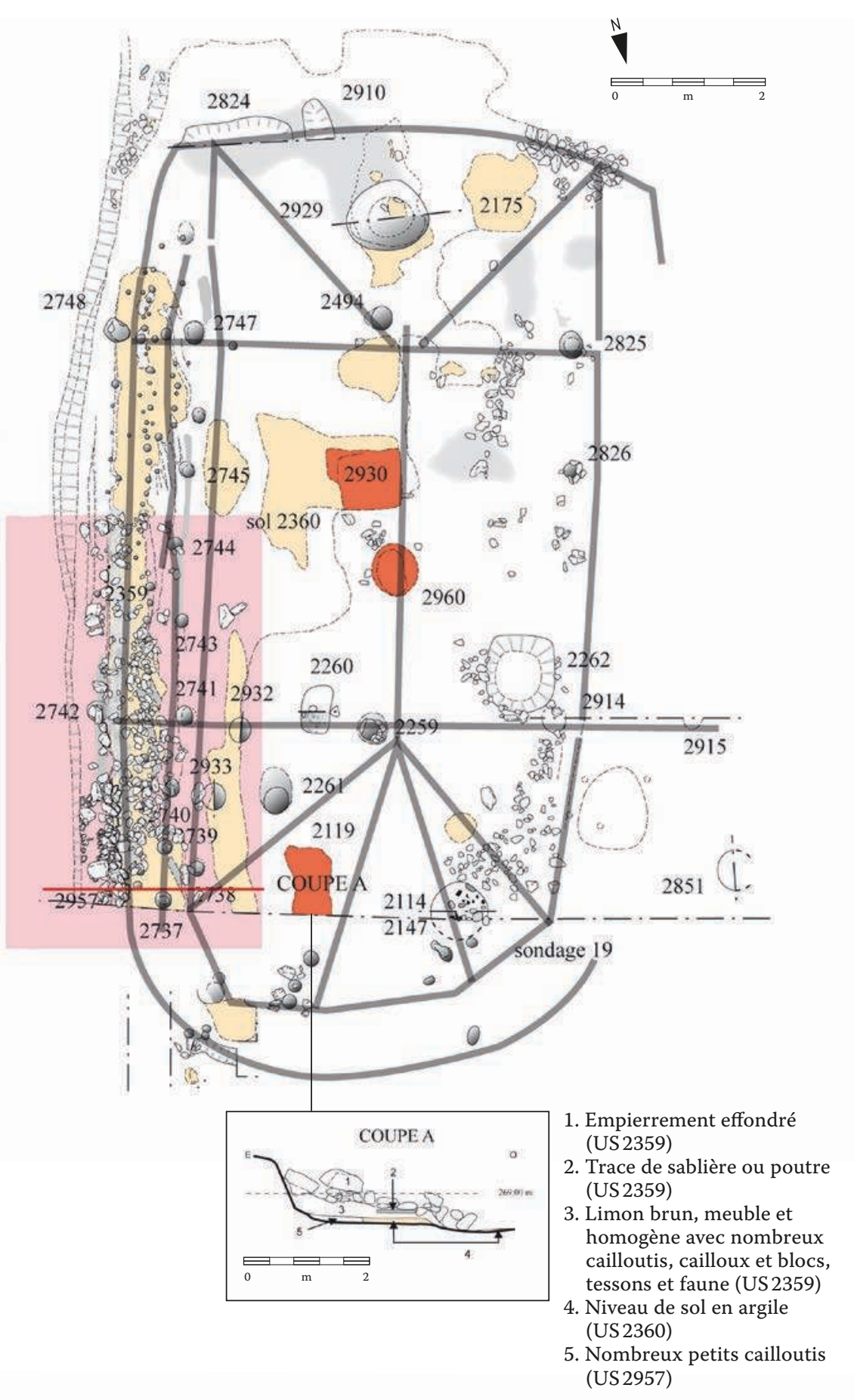


longitudinaux : on dresse d'abord les alignements de poteaux porteurs coiffés de la faîtière et des sablières, puis les chevrons disposés librement viennent réunir les trois ou cinq lignes de supports (Buchsenschutz, 2005). Cependant, il est préférable de relier en premier les têtes de poteaux par l'entrait avant de mettre en place les chevrons. Un prolongement d'un mètre vers l'est de ces deux tierces structurelles nord et sud est matérialisé par deux poteaux supplémentaires. Le pendant de ce prolongement à l'ouest n'est pas attesté, sauf par un seul trou de poteau dans l'axe du portique nord, mais qui semble un peu trop éloigné. Au nord de cette travée, une abside ferme le bâtiment, comme dans la plupart des édifices mis au jour sur le site, tandis qu'au sud une travée égale à la moitié de la travée centrale pourrait soit constituer une toiture en pavillon, soit former un " porche » couvert. La façade de cette travée sud est matérialisée plus particulièrement, au sud-ouest, par le reste d'un angle de muret en pierre sèche : il s'agirait là du vestige ténu d'un aménagement lié au terrassement de nivellement du bâtiment, le sol en argile nécessitant d'être légèrement surélevé à l'ouest et au sud-ouest, tout comme sa mise en place nécessitait d'entailler la pente de la combe à l'est. Il est difficile d'établir un phasage entre les différents éléments clôturant l'espace interne de l'édifice : une succession de parois est visible à l'est. Le premier mur est matérialisé par une tranchée d'une vingtaine de centimètres de largeur, limitant le sol en argile : ponctionnée de petits trous de piquets sur son côté ouest, elle montre aussi des traces de bois sur solin en cailloutis dans sa partie nord. On peut envisager l'hypothèse d'une première paroi sur sablière basse calée par les piquets dont la présence sur le côté ouest serait peut-être liée aux problèmes de stabilisation du terrain (Buchsenschutz, 2005). La seconde séparation, située à une cinquantaine de centimètres à l'ouest de la précédente, est constituée d'une ligne irrégulière de petits trous de piquets, servant sans doute de support à un clayonnage : il n'est pas exclu qu'il s'agisse là d'un élément non structurel (comme une banquette, par exemple, ce qui est proposé notamment à Sorgenti della Nova en Italie, pour des bâtiments datés du Bronze final/début de l'âge du Fer, Domanico, 2005). Enfin, une série de poteaux s'alignent entre les poteaux latéraux des tierces nord et sud, avec des vestiges d'empreintes de bois les reliant: s'agit-il là encore de parois sur sablières ? Ces parois sont-elles toutes contemporaines, ou participentelles de différents remaniements, peut-être en relation avec des problèmes successifs de poussée, de ruissellement et de colluvionnement liés à la pente du terrain ? Qu'elles fonctionnent en même temps reste cependant une hypothèse envisageable. À Antran dans la Vienne, une hypothèse a été proposée pour les trois parois successives : poteaux internes supportant la charpente, puis piquets de soutien d'un clayonnage formant la paroi du bâtiment, puis poteaux de support de la rive de toiture débordante. Cette configuration pourrait s'appliquer au bâtiment 4 (Pautreau, 1988). On sait cependant qu'un deuxième état du bâtiment existe, puisque les tranchées et le sol en argile sont dans un deuxième temps recouverts à l'est par une sablière posée contre un muret de pierre sèche, lequel sert de soutènement (et de drainage ?) à l'entaille du substrat.

Trois échantillons micromorphologiques ont été prélevés dans les dépôts liés à cette occupation hallstattienne. Deux d'entre eux recoupent le revêtement de sol argileux et la couche qui le recouvre. Leur étude a mis en évidence la présence de nombreux témoins de combustion (charbons, os et fragments de sol brûlés) dans la couche surmontant le placage d'argile. Ce dernier correspond plus précisément à un amalgame d'argile gris-jaune comparable à celui du revêtement de sol du bâtiment 3 . Il repose sur un horizon pédologique défriché par brûlis. Le sol du bâtiment semble avoir été refait à plusieurs reprises, par l'apport de nouvelles chapes argilocarbonatées piégeant des résidus d'occupation tels que cendres, charbons ou fragments de poterie. Dans la couche sus-jacente (2114), l'abondance des charbons, des agrégats argilo-humiques carbonisés et des fragments de mortier argilo-micritique suggère la destruction par le feu d'une structure en bois et en terre.

\section{Le bâtiment 5}

Au centre du village, un bâtiment avait une structure et une fonction particulière [ill. 6]. Cet édifice, identifiable comme un atelier, est très difficilement restituable en détail. Une bipartition en deux travées proche du carré apparaît nettement : la moitié sud, caractérisée par les murets de pierres sèches et par deux poteaux, serait en grande partie abritée par des parois pleines, alors que la moitié nord, plus légère, pourrait être ouverte sur les côtés. Dans chaque espace se trouve un foyer. Au sud, le foyer, non aménagé, est posé sur une couche cendreuse et charbonneuse, tandis que le foyer situé au nord est bordé par trois pierres sur chant au milieu desquelles passait une tuyère. Son aire de chauffe est constituée d'un radier de petites pierres sur lesquelles est plaquée une couche d'argile cuite formant la sole qui repose sur un sol en argile jaune. Lors de la fouille, de nombreuses battitures et coulées de bronze ont été recueillies à proximité de ce dernier, démontrant qu'à cet endroit étaient travaillés aussi bien le fer que les alliages cuivreux.

\section{Le bâtiment 6}

Le bâtiment 6 n'est conservé que sur un tiers de sa surface, vers l'est [ill. 7]. Il présente un plan à abside au nord. Un solin en cailloutis et des traces de bois, sur une largeur de $15 \mathrm{~cm}$ à $20 \mathrm{~cm}$, délimitent le sol en argile. Au sud de l'édifice, l'angle est de sa façade est marqué par trois 


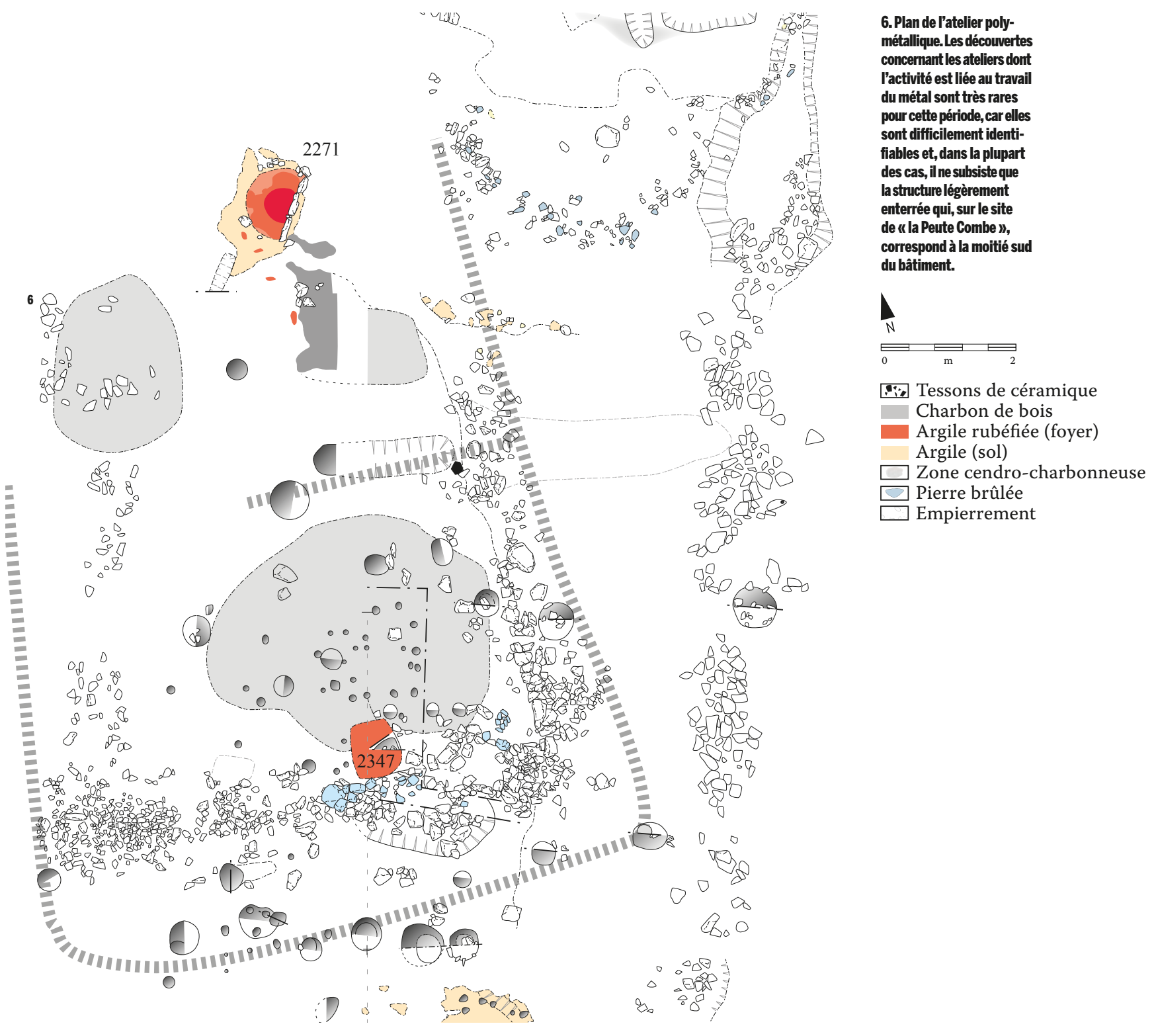

poteaux. Deux d'entre eux forment à l'évidence des poteaux corniers, le troisième, plus à l'ouest, pourrait constituer le montant de la porte axiale (largeur de passage de l'ordre de $80 \mathrm{~cm}$ ) du bâtiment, si l'on applique un principe simple de symétrie. On peut d'ailleurs tenter de restituer l'emplacement de la paroi ouest par symétrie. La structure porteuse interne pourrait être matérialisée par une paroi au nord (large saignée dans le sol en argile [US 2434], trous de poteaux à l'est complétés éventuellement par un poteau symétrique à l'ouest assurant la présence d'un portique), un portique à deux poteaux au sud, et entre les deux, un poteau central soulageant peutêtre la faîtière. Le plan et la structure rappellent ceux de Nola (Livadie, 2005, maison 4) car nous avons en effet ici un bâtiment à abside, de 5,50 $\mathrm{m}$ de large pour 12,5 $\mathrm{m}$ de long. Les trois divisions internes se retrouvent dans la maison de Nola, avec notamment un cloisonnement plus « épais » juste avant l'abside, comme on pourrait l'envisager ici. Il n'est pas exclu par ailleurs que, comme à Nola, les rares poteaux internes n'aient joué qu'un rôle mineur «d'accompagnement » de la structure porteuse, le mur périphérique assurant la stabilité de l'ensemble (Livadie, 2005).

\section{Le bâtiment 7}

Le bâtiment 7, de $7 \mathrm{mx}$ 13,5 m, présente sans doute deux états [ill. 8]. Le premier voit la mise en place d'un sol en argile, délimité par des parois dont la nature reste indéterminée : aucune trace de celles-ci n'ayant été retrouvée, leur restitution s'appuie sur des effets de parois le long du sol en argile et de l'empierrement (muret de soutènement?) à l'est, ainsi que sur la présence de pierres de calage et d'un cloisonnement interne. Une abside, marquée par un alignement ténu de pierres, fermerait ce premier bâtiment au nord. On voit que la plupart des bâtiments du site présentent au 

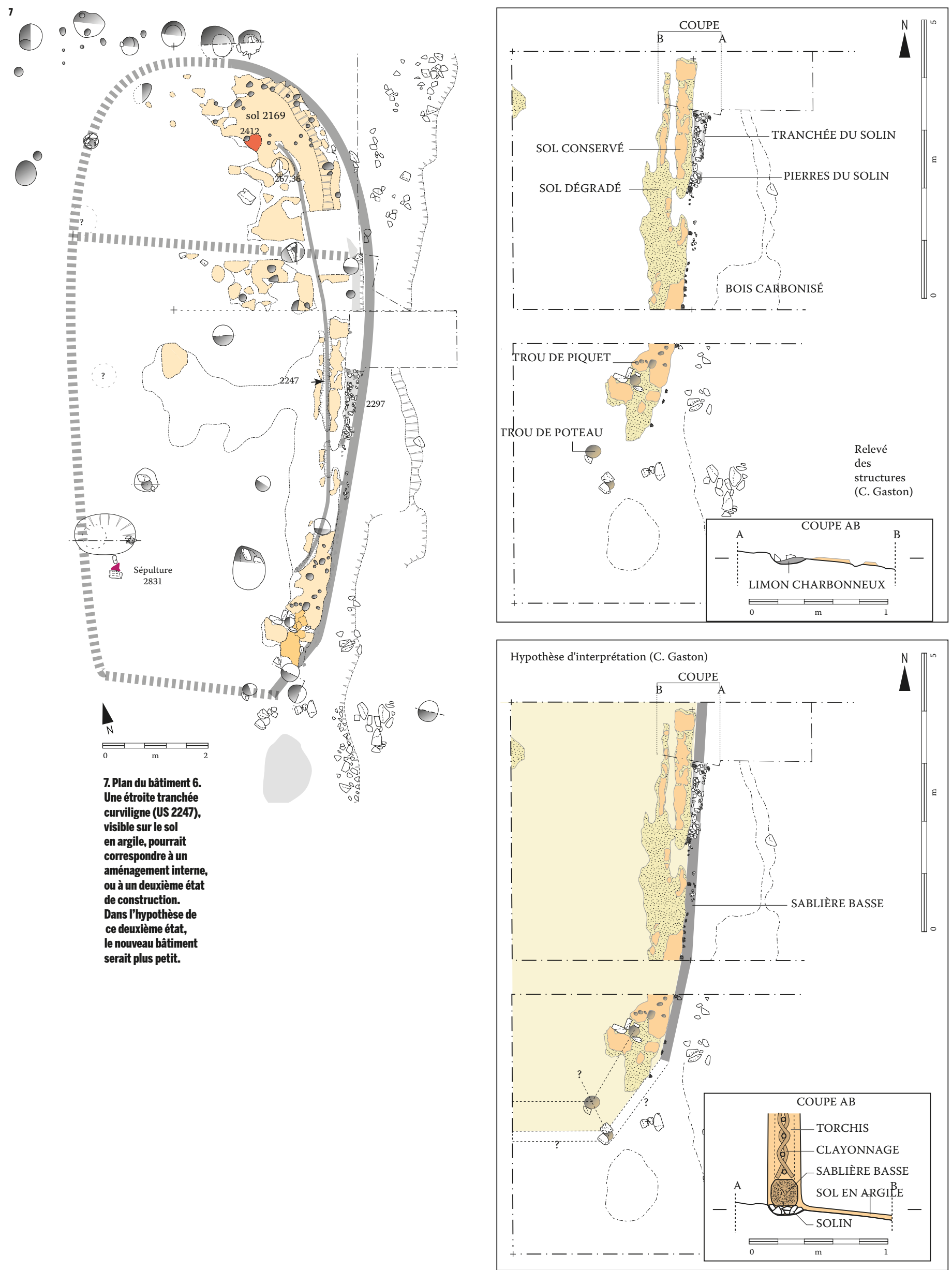


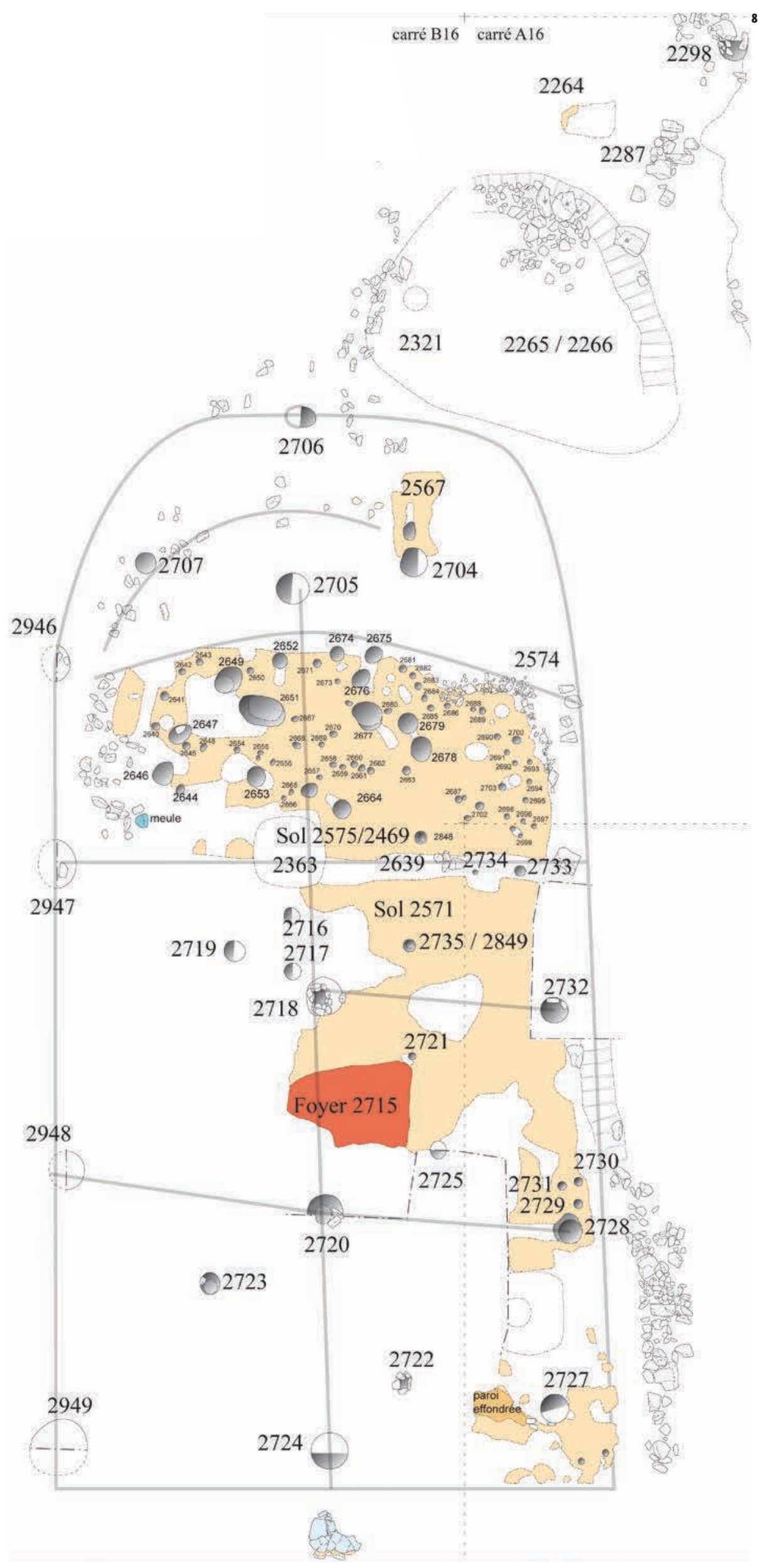

8. Plan du bâtiment

7. Une série de trous de

piquets et de poteaux

ainsi qu'une tranchée

transversale (US 2639)

percent le sol,

matérialisant une

structure à abside.

$\Delta$

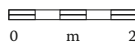

Tessons de céramique

Charbon de bois

Argile rubéfiée (foyer)

Argile (sol)

Zone cendro-charbonneuse

Pierre brûlée

Empierrement 
moins une abside. La structure de celui-ci est un châssis de bois à trois travées, la travée centrale, elle-même subdivisée en deux dans sa moitié est, abritant le foyer. Une paire de poteaux plus rapprochés semble constituer un renforcement de la faîtière au nord, avant le passage à l'abside supposée. La mise en place d'un nouveau sol en argile, visible au nord-est de l'abside, marque un réaménagement, peut-être limité à l'abside, de l'espace interne du bâtiment.

\section{Les autres traces de sols en argile sur le site}

Plusieurs sols en argile ont été observés à divers endroits sur le site, mais, comme ils sont très altérés, ils n'apportent que peu de renseignements sur les structures auxquelles ils sont liés.

Le bâtiment 8 n'est délimité que par son creusement dans le substrat à l'est. Le plan ovalaire est donc très hypothétique, tout comme la dimension en largeur de l'édifice. Ce qui est assuré, c'est la présence de deux états de sol: d'abord un sol en argile, puis la mise en place d'un plancher, comme l'indiquent les encastrements de lambourdes, ce qui est exceptionnel, puisque la présence de planchers dans les bâtiments reste rare pour cette période.

Le bâtiment 12 n'est conservé que sur le quart nord-est de sa surface. En effet, il pourrait être bâti selon un plan circulaire ou ovalaire, comme le suggère le tracé curviligne du lambeau de sol argileux conservé, et la position centrale du foyer mis en évidence : dans ce cas de figure, ses dimensions seraient de l'ordre de $5 \mathrm{~m}$ de large pour $6 \mathrm{~m}$ de long. Sa fonction reste imprécise, mais il pourrait être lié au bâtiment 4 dans la deuxième phase de celui-ci.
Dans le bâtiment 13, fortement détruit par un puits gallo-romain, la seule trace d'un sol en argile jaune a été mise en évidence près de la seconde plaque foyère dont l'activité était liée à la métallurgie et la forme rappelait celle découverte dans la partie nord de la forge.

Les découvertes de sol en argile sur les sites protohistoriques sont très rares et le plus souvent peu décrites ou peu publiées, ce qui rend les comparaisons difficiles. Les analyses des lames minces déjà réalisées nous renseignent sur la nature et la mise en place de ces sols et montrent une uniformité dans la fabrication de ces chapes argileuses et dans la manière de les poser sur un sol défriché et épierré. La présence d'interruptions correspondant à des sablières basses démontre que la réalisation du sol est effectuée en fin de construction, lorsque l'armature principale du bâtiment est en place. Des rechapages sont effectués à plusieurs reprises, au moins dans le bâtiment 4. La présence de piquets ou de poteaux secondaires traversant le sol indique en revanche qu'il est antérieur à l'aménagement interne des cloisons. Par manque de comparaisons avec d'autres sites de la même période, ces premières interprétations restent limitées. Les analyses complémentaires sur les lames minces non encore étudiées devraient apporter des renseignements sur les constructions mixtes associant terre crue et bois. L'absence de phytolithes qui amène à écarter l'hypothèse de cloisons en torchis et de toits de chaume sera peut-être remise en question. éditions du CTHS, p. 267-297.

Labeaune R., Alix S. (DIR.), 2014, Talant, Plombièresles-Dijon, Côte-d'Or, Bourgogne, Peute Combe, les Vaux Bruns. Découvertes d'un établissement rural gallo-romain et d'un hameau à vocation artisanale $d u v^{e}$ siècle avant J.-C., rapport d'opération, Inrap-

BuCHEZ N., 2005 "Architecture de l'habitat de lâge du Bronze à La Tène ancienne dans la Somme », in Buchsenschutz O., Mordant C., Architectures protohistoriques en Europe occidentale du Néolithique final à lâge du Fer, Actes du $127^{\mathrm{e}}$ congrès de Nancy (15-20 avril 2002), Paris, éditions du CTHS, p. 203-208.

BUCHSENSCHUTZ O., 2005, « Du comparatisme à la théorie architecturale », in BuCHSENSCHUTZ O., Mordant C., Architectures protohistoriques en Europe occidentale du Néolithique final à l'âge du Fer, Actes du $127^{\mathrm{e}}$ congrès de Nancy (15-20 avril 2002), Paris, éditions du CTHS, p. 49-63.

Curdy P. et al., 1993, « Brig-Glis/Waldmatte, un habitat alpin de l'âge du Fer, fouilles archéologiques N9 en Valais », Archéologie Suisse, 16, p. 138-151.

DOMANICO L., 2005, « Tradition et innovation dans l'architecture de lâge du Bronze final et du premie âge du Fer en étrurie », in BuCHSENSCHutz O.,
SRA Bourgogne, 6 vol. (418, 482, 29, 186, 519, 374 p.).

LIVADIE C.-A. et al., 2005, «Sur l'architecture des cabanes du Bronze ancien final de Nola (NaplesItalie) ", in Buchsenschutz O., Mordant C., Architectures protohistoriques en Europe occidentale du Néolithique final à l'âge du Fer, Actes $\mathrm{du} 127^{\mathrm{e}}$ congrès de Nancy (15-20 avril 2002), Paris, éditions du CTHS, p. 487-512.

Pautreau J.-P., 1988, « La Croix-Verte à Antran (Vienne) », in Audouze F., Buchsenschutz O. Architecture des âges des métaux: fouilles récentes, Paris, Errance, « Archéologie Aujourd'hui, Dossiers de protohistoire $\mathrm{n}^{\circ} 2 »$, p. 47-53.

Sordoillet D., 2009, Géoarchéologie de sites préhistoriques. Le Gardon (Ain), Montou (Pyrénées orientales), et Saint-Alban (Isère), Paris, éd. de la Maison des Sciences de l'Homme, « Documents d'archéologie française, 103 », 188 p. 